\title{
Hanford Site Performance Summary - EM Funded Programs August 1995
}

\author{
E. A. Schultz
}

Date Published

August 1995

Prepared for the U.S. Department of Energy

Office of Environmental Management

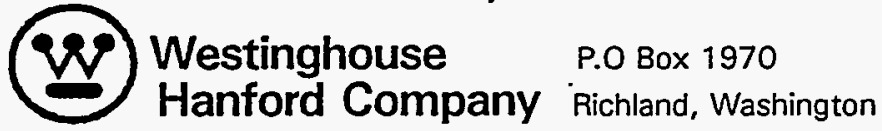

Management and Operations Contractor for the

U.S. Department of Energy under Contract DE-AC06-87RL10930

Approved for Public Release 


\section{DISCLAIMER}

Portions of this document may be illegible in electronic image products. Images are produced from the best available original document. 
LEGAL DISCLAIMER

This report was prepared as an account of work sponsored by an agency of the United States Government. Neither the United States Government nor any agency thereof, nor any of their employees, nor any of their contractors, subcontractors or their employees, makes any warranty, express or implied, or assumes any legal liability or responsibility for the accuracy completeness, or any third party's use or the results of such use of any information, apparatus, product, or process disclosed, or represents that its use would not infringe privately owned rights. Reference herein to any specific commercial product, process, or service by trade name, trademark, manufacturer, or otherwise, does not necessarily constitute or imply its endorsement, recommendation, or favoring by the United States Government or any agency thereof or its contractors or subcontractors. The views and opinions of authors expressed herein do not necessarily state or reflect those of the United States Government or any agency thereof.

This report has been reproduced from the best available copy. Available in paper copy and microfiche.

Available to the U.S. Department of Energy and its contractors from

Office of Scientific and Technical Information P.O. Box 62

Oak Ridge, TN 3783

(615) $5 \% 6-8401$

Available to the public from the U.S. Department of Commerce National Technical Information Service

5285 Port Royai Road

Springfield, VA 22161

(703) $487-4650$

Printed in the United States of Americe

DISCLM-1.CHP (1-91) 


\section{RELEASE AUTHORIZATION}

Document Number: $\quad W H C-S P-0969-53$

Document Title: Hanford Site Performance Summary - EM Funded Progarms

Release Date: $\quad 10 / 10 / 95$

This document was reviewed following the procedures described in WHC-CM-3-4 and is:

\section{APPROVED FOR PUBLIC RELEASE}

WHC Information Release Administration Specialist:

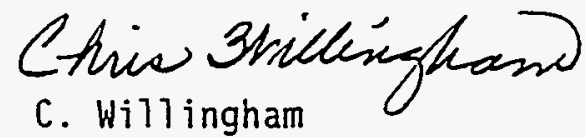

C. Willingham

$10 / 10 / 95$

- ^GMACPATH ${ }^{\sim}$ GEF1000^G 


\section{HANFORD SITE PERFORMANCE SUMMARY - AUGUST 1995}

Performance data for August 1995 reflects a four percent unfavorable schedule variance ( $\$ 65.9$ million*) and is an increase over July 1995 ( $\$ 65.9$ million for August versus $\$ 58.2$ million for July). The majority of the behind schedule condition is attributed to EM-30, (Office of Waste Management), specifically the Tank Waste Remediation System (TWRS) Program, and is a result of external delays in receiving key decision 0 (KD-0) for Project W-314, "Tank Farm Restoration and Safe Operations" and approval of the Multi-Waste Tank Facility basel ine change request $(B C R)$. A breakdown of individual program performance is 1 isted on page 16 .

The TWRS schedule variance $(-\$ 55.2$ million) is attributed to the MWTF workscope still being a part of the baseline ( $\$ 44.5$ million). A BCR is in process deleting the MUTF from the TWRS baseline. Once the BCR is approved and implemented, the overall schedule variance will be reduced to $\$ 21.4$ million.

Eighty-one enforceable agreement milestones were scheduled FYTD. Seventy-five (93 percent) of the eighty-one were completed on or ahead of schedule, two were completed 7ate - M-45-07B, "Reach Decision on Whether to Proceed with Demonstration" and M-15-10C, "100-KR-1 Operable Unit (OU) Focused Feasibility Study and Interim Remedial Measure (IRM)") - and four are delinquent M-43-02A, "W-314B Doubie-She 71 Tank Ventilation Upgrades Conceptual Design Report (CDR)"; M-43-04A, "W-314A Tank Farm Instrumentation Upgrades CDR"; M-17-14, "Injtiate Operations - 200 Area Effluent Treatment Facility"; and M-17-29, "Implement Best AvailabTe Technology/A17 Known, Available, and Reasonable Methods of Prevention, Control and Treatment (BAT/AKART) for 242-A Evaporator Process Condensate Stream." Tri-Party Agreement milestones $M-43-02 A$ and $M-43-04 A$ belong to the TWRS Program and are associated with the delay in KD-0 for Project W-314. Tri-Party Agreement milestones M-17-14 and M-17 29 belong to the Liquid Waste Program and were impacted by the delay in the 200 Area Effluent Treatment Facility. Additional information on these milestones can be found on pages 26 through 28 .

Performance data reflects a continued significant favorable cost variance of $\$ 147.8$ million (10 percent). The cost variance is attributed to process improvements/efficiencies, elimination of low-value work, and workforce reductions and is expected to continue for the remainder of this fiscal year. A small portion of the cost variance is attributed to a delay in billings which should self-correct by fiscal year-end.

*Dollar figures include all fund types - expense, capital equipment not related to construction, and construction. Data is derived from the office of Environmental Restoration and Waste Management's Progress Tracking System. 


\section{WHC-SP-0969-53}




\section{HANFORD EM STATUS BY CONTROL POINT \\ - All Fund Types - \\ (August 1995)}

$+$

EM 10

EM 20

$\omega$

EM 30

EM 40

EM 50

EM 60

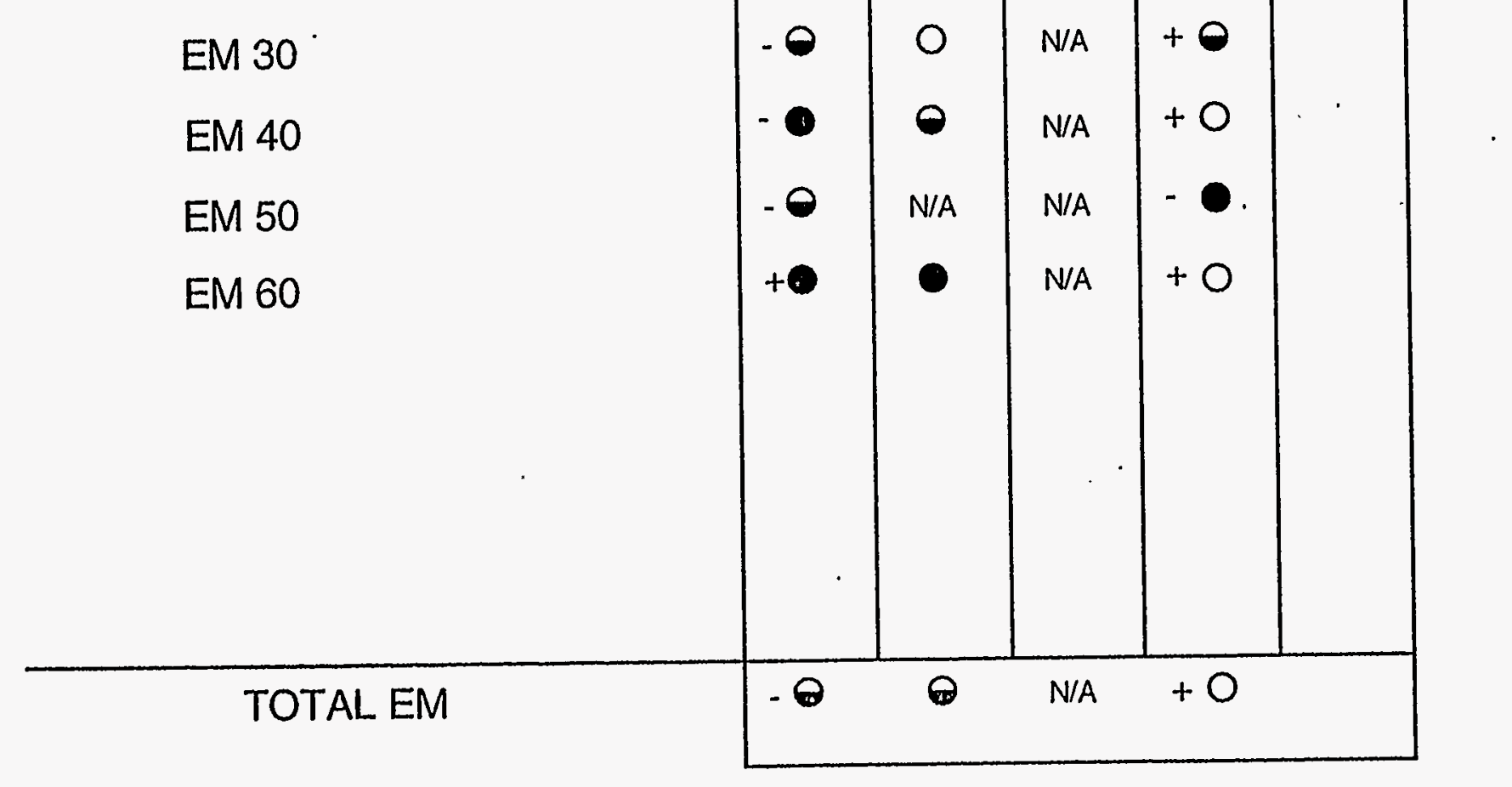

Level of

Management

Action Needed:

Satisfactory

Minor Concern

Major Concarn

ENFORCEABLE AGREEMENT MILESTONES

Achieving all Milestones

$<10 \%$ of milestones no more than 6 months late)

$>10 \%$ of milestones more than 6 months late)

\section{COST/SCHEDULE}

Cost/schedule as planned $(<+1-3 \%)$

C cost/schedule $>+1-3 \%<+1-10 \%$

Cost/schedule $>+1-10 \%$ 


\section{EM COST PERFORMANCE - ALL FUND TYPES}

AUGUST 1995

(\$ In Millions)

\begin{tabular}{|c|c|c|c|c|c|c|c|}
\hline & BCWS & $\begin{array}{l}\text { FYTD } \\
\text { BCWP }\end{array}$ & ACWP & SV & CV & $\begin{array}{c}\text { FY } \\
\text { BUDGET }\end{array}$ & $\begin{array}{l}\text { BCWS } \\
\text { CHANGE FROM } \\
\text { PRIOR MONTH }\end{array}$ \\
\hline EM 10 & 2.4 & 2.3 & 2.7 & $(0.1)$ & $(0.4)$ & 2.4 & 0.0 \\
\hline EM 20 & 20.5 & 17.2 & 15.4 & (3.3) & 1.8 & 26.4 & 1.7 \\
\hline EM 30 & 958.2 & 899.4 & 821.4 & (58.8) & 78.0 & $1,106.3$ & (1.7) \\
\hline EM 40 & 201.9 & 198.2 & 158.3 & (3.7) & 39.9 & 252.0 & (10.2) \\
\hline EM 50 & 42.6 & 39.8 & 40.2 & (2.8) & $(0.4)$ & 50.0 & 1.1 \\
\hline EM 60 & 268.5 & 271.3 & 242.4 & 2.8 & 28.9 & 305.0 & $(7.7)$ \\
\hline TOTAL EM & $1,494.1$ & $1,428.2$ & $1,280.4$ & (65.9) & 147.8 & $1,742.1$ & (16.8) \\
\hline
\end{tabular}


WHC-SP-0969-53

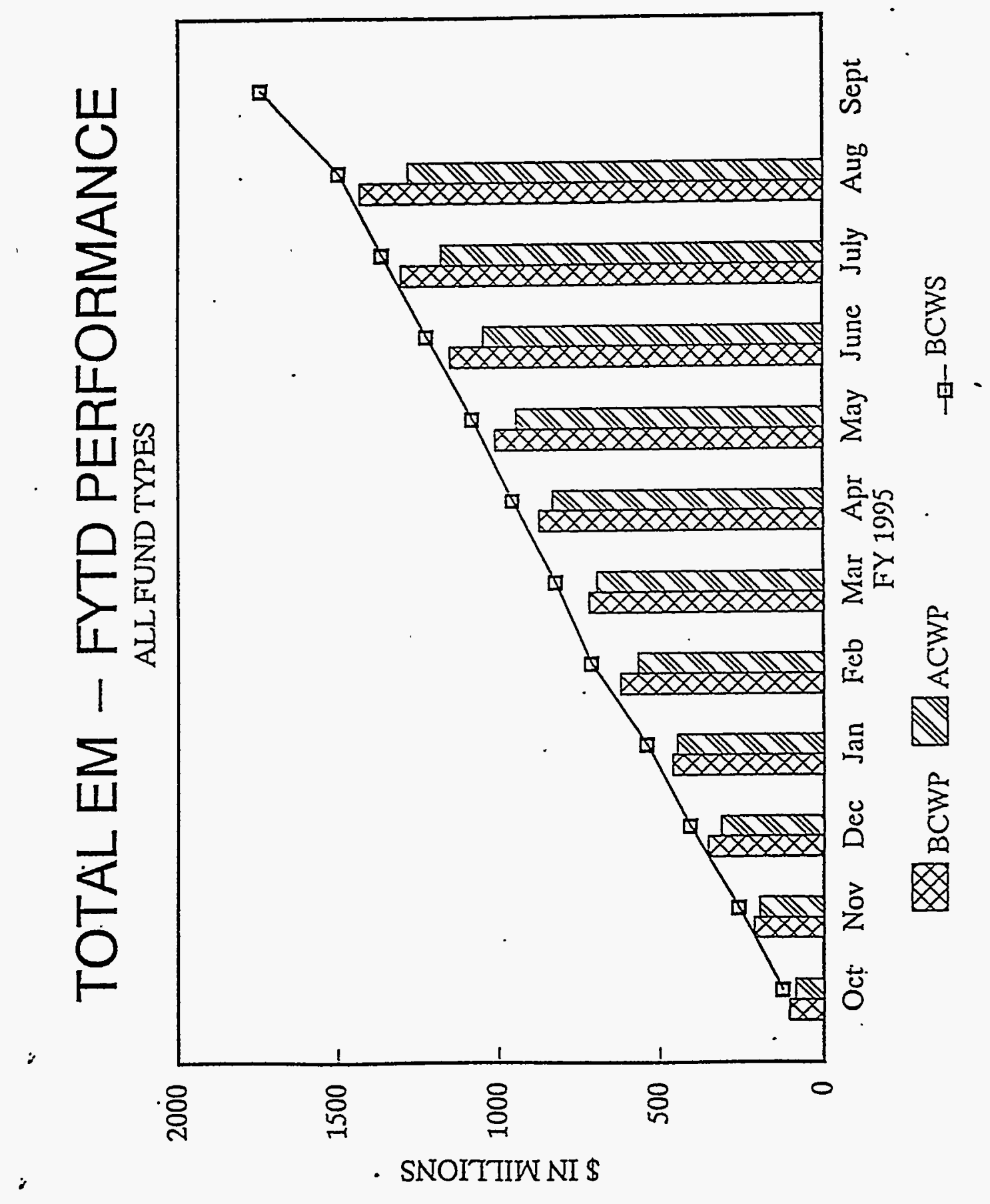




\section{HANFORD EM STATUS BY WBS \\ - All Fund Types - \\ (August 1995)}

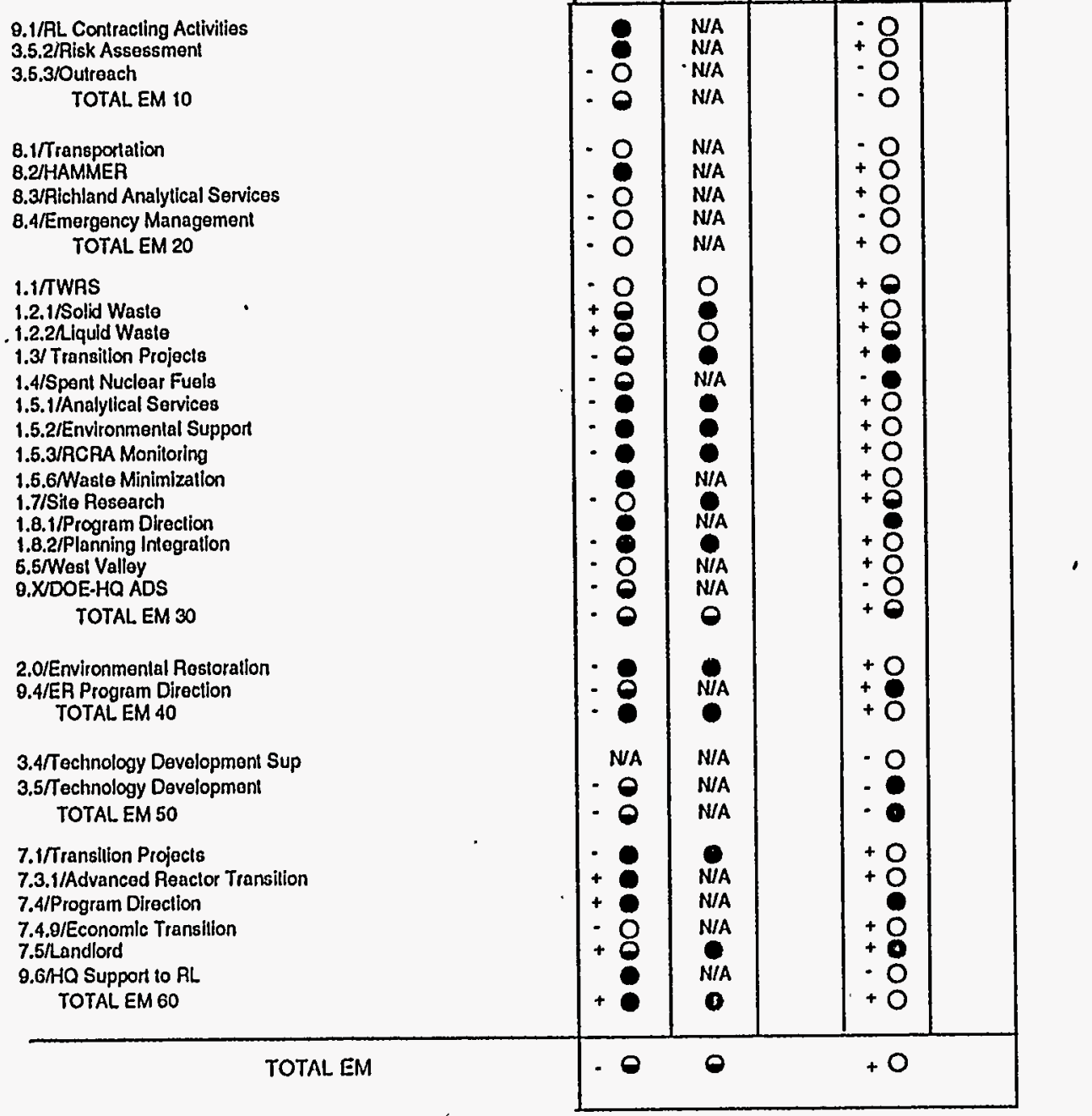

LEVEL OF MANAGEMENT ACTION NEEDED:

Satslactory

O Minor Concom

O Major Concem

ENFORCEABLE AGREEMENT

Achioving all Milostonos

$<10 \%$ of milostonos no more than 6 monins lalo

$O>10 \%$ of milostonos mor
than 6 monlhs lato

COST/SCHEDULE

Cosvschodulo as plannod (< $+1-3 \%)$

Cosyschadulo $>+1.3 \%<+1.10 \%$

O costschodule $>+1.10 \%$

- Nogallvo Varianco + Posillvo Varlance 

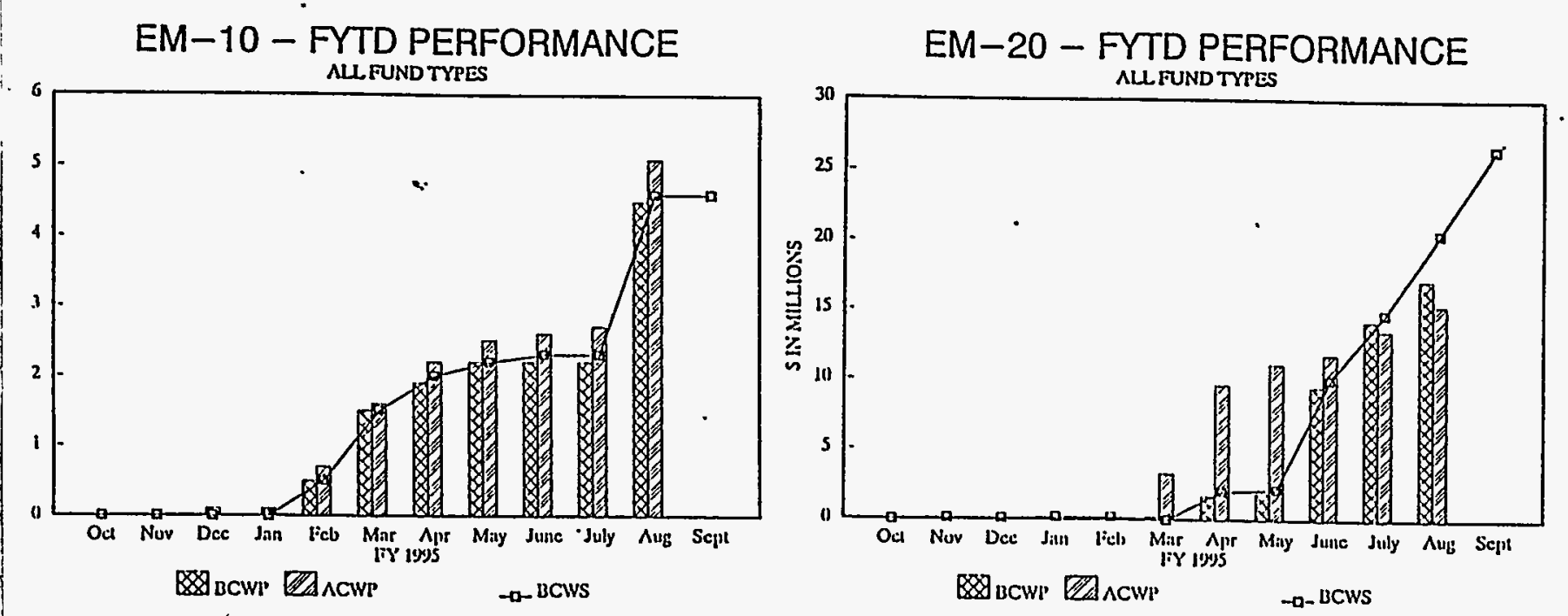

$\infty$

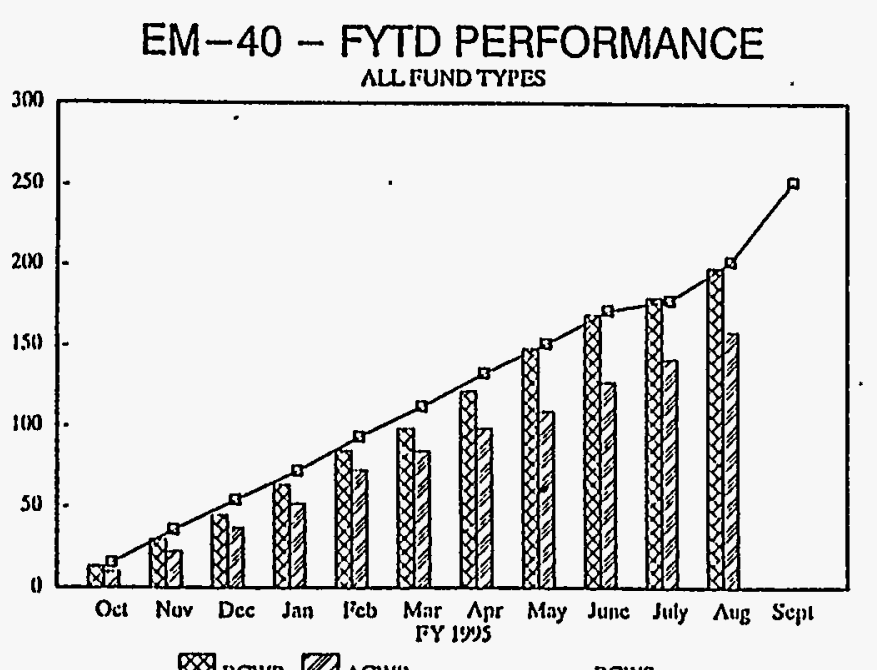

BCIVP

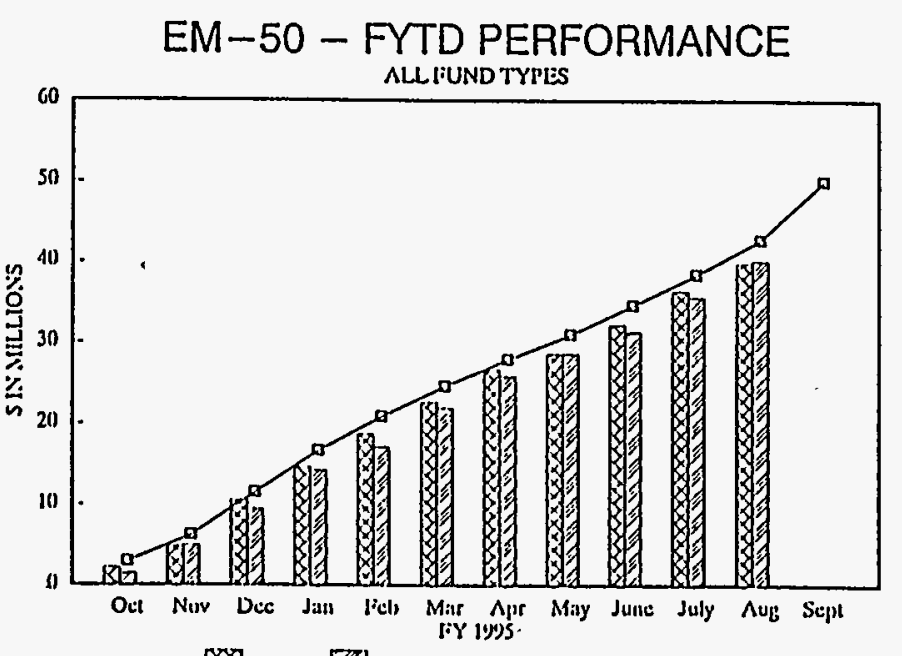

BCW!
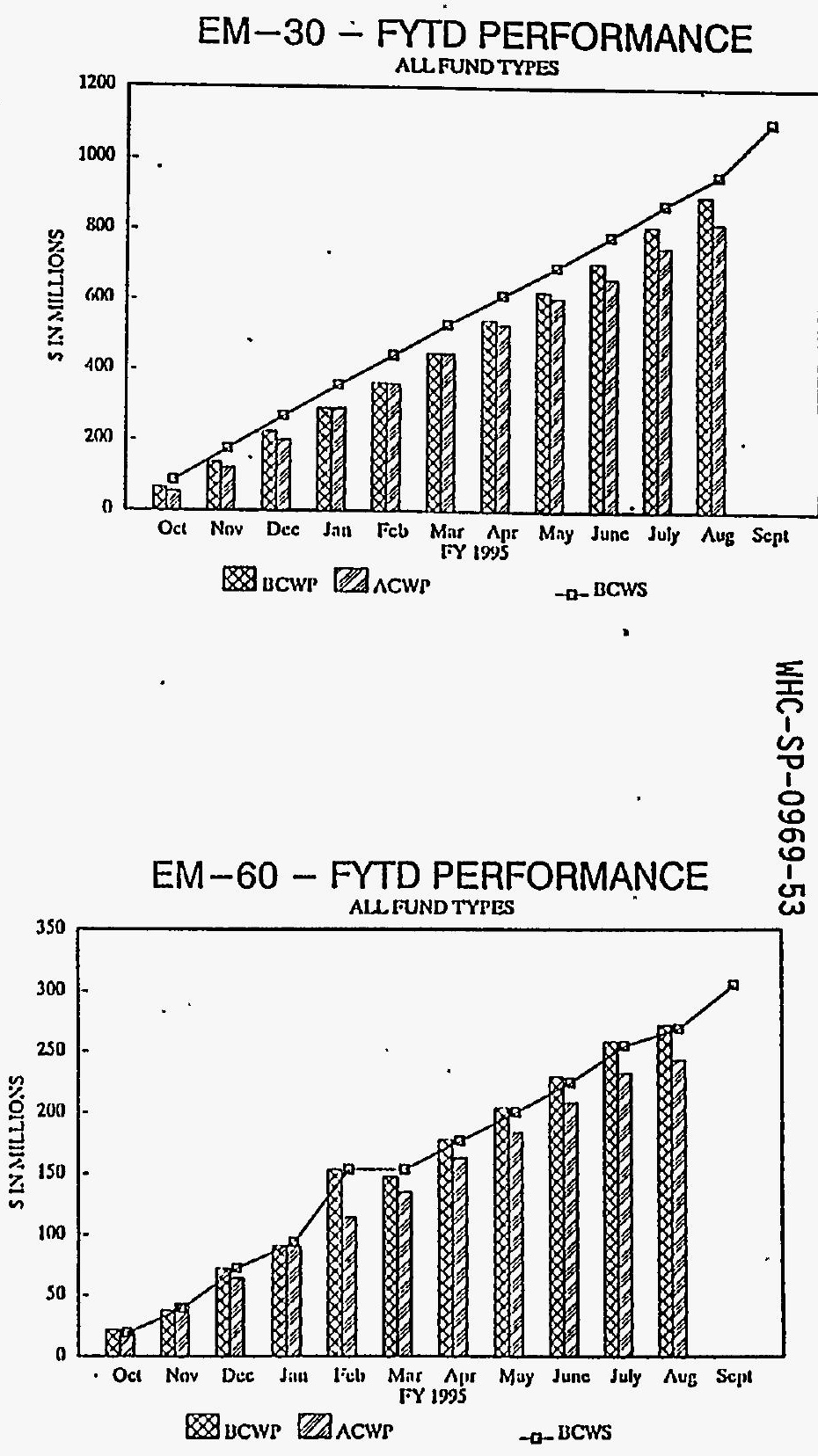


\section{EM 10 Cost/Schedule Summary \\ Total \$}

FYTD BCWS MS's Cost/Schedule Through August 1995

9.1 RL Contracting Activities

3.5.2 Risk Assessment

3.5.3 Outreach

$\infty$
2.1

0.2

0.1

2.4

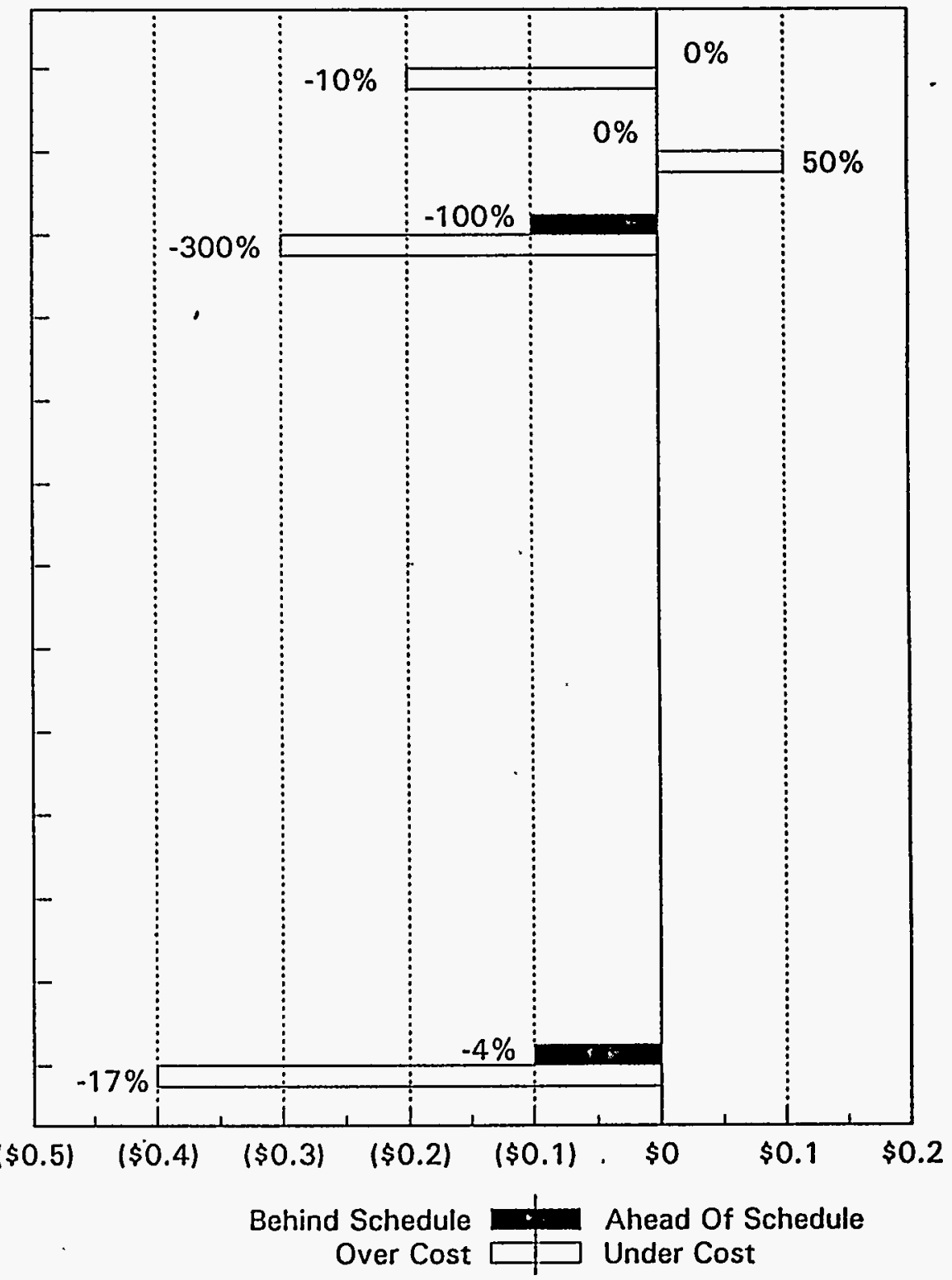




\section{EM 20 Cost/Schedule Summary \\ Total \$}

8.1 Transportation

8.2 HAMMER

8.3 Richland Analytical Services

8.4 Emergency Management

๘
FYTD BCWS M\$'S

5.0

12.7

2.6

0.2

20.5

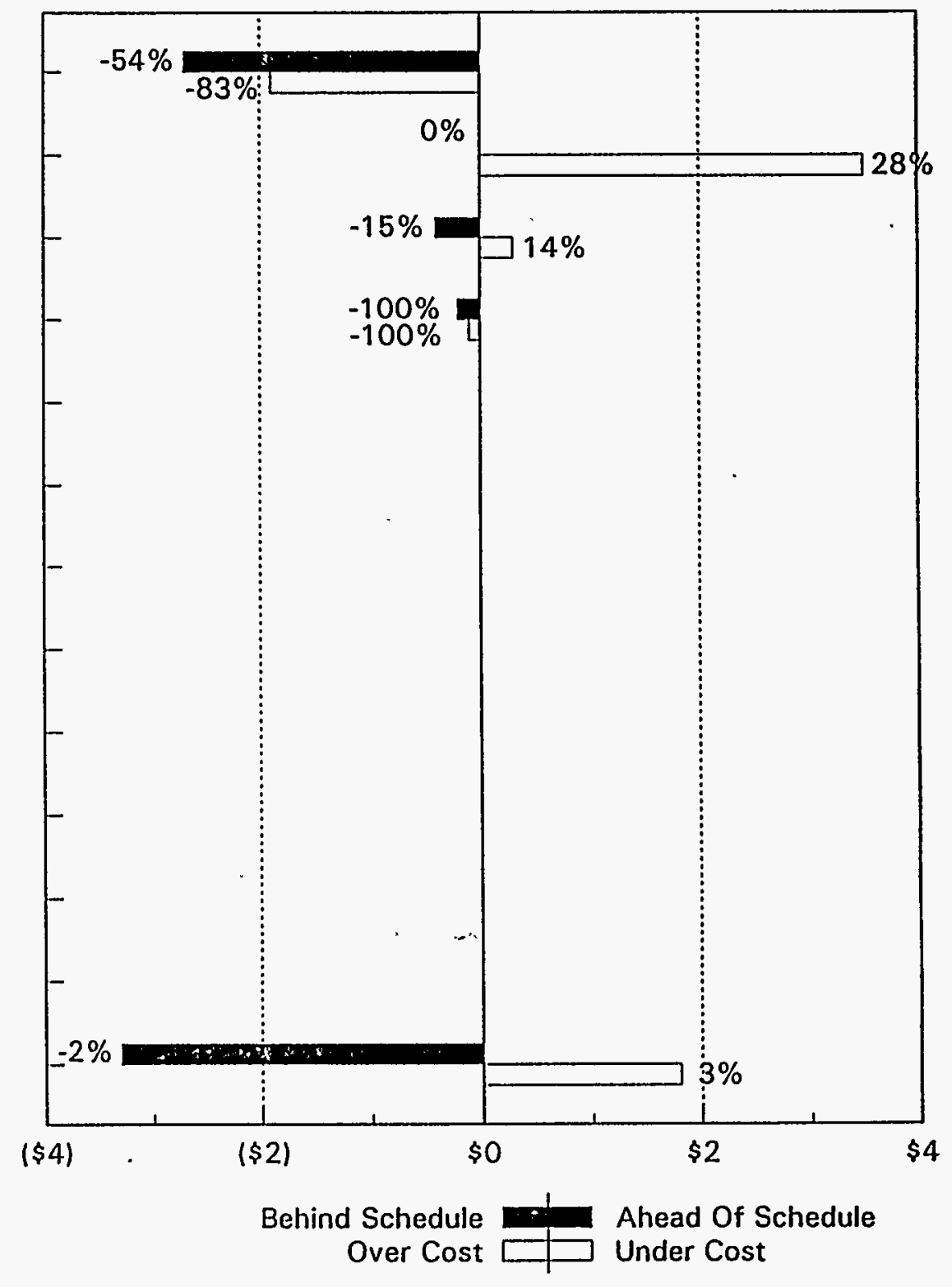




\section{EMI 30 Cost/Schedule Summary \\ Total \$}

\section{FYTD BCWS M\$s's Cost/Schedule Through August 1995}

1.1 Tank Waste Remediation System

1.2.1 Solid Waste

1.2.2 Liquid Waste

1.3.1 Facility Operations

1.4 Spent.Nuclear Fuels

1.5.1 Analytical Services

$\varpi$

1.5.3 RCRA Monitoring

1.5.6 Waste Minimization

1.7 Science \& Tech Research

1.8.1 RL Program Direction

1.8.2 Planning Integration

5.5 West Valley

9.X DOE-HO ADS

Total EM 30
528.3

87.6

52.1

31.4

78.6

49.4

8.7

25.7

0.5

41.8

28.0

13.0

2.9

10.2

958.2

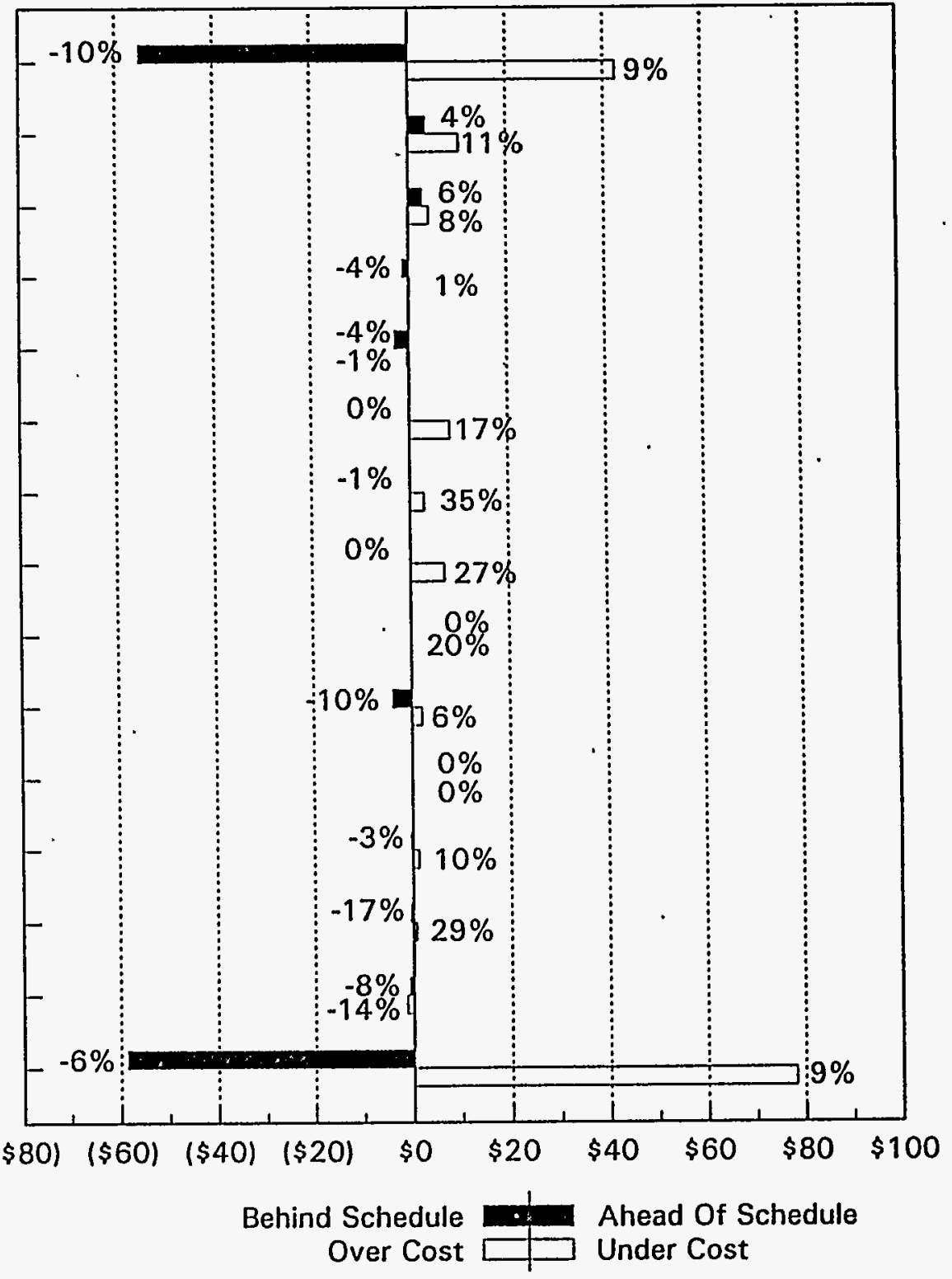




\section{EM 40 Cost/Schedule Summary \\ Total \$}

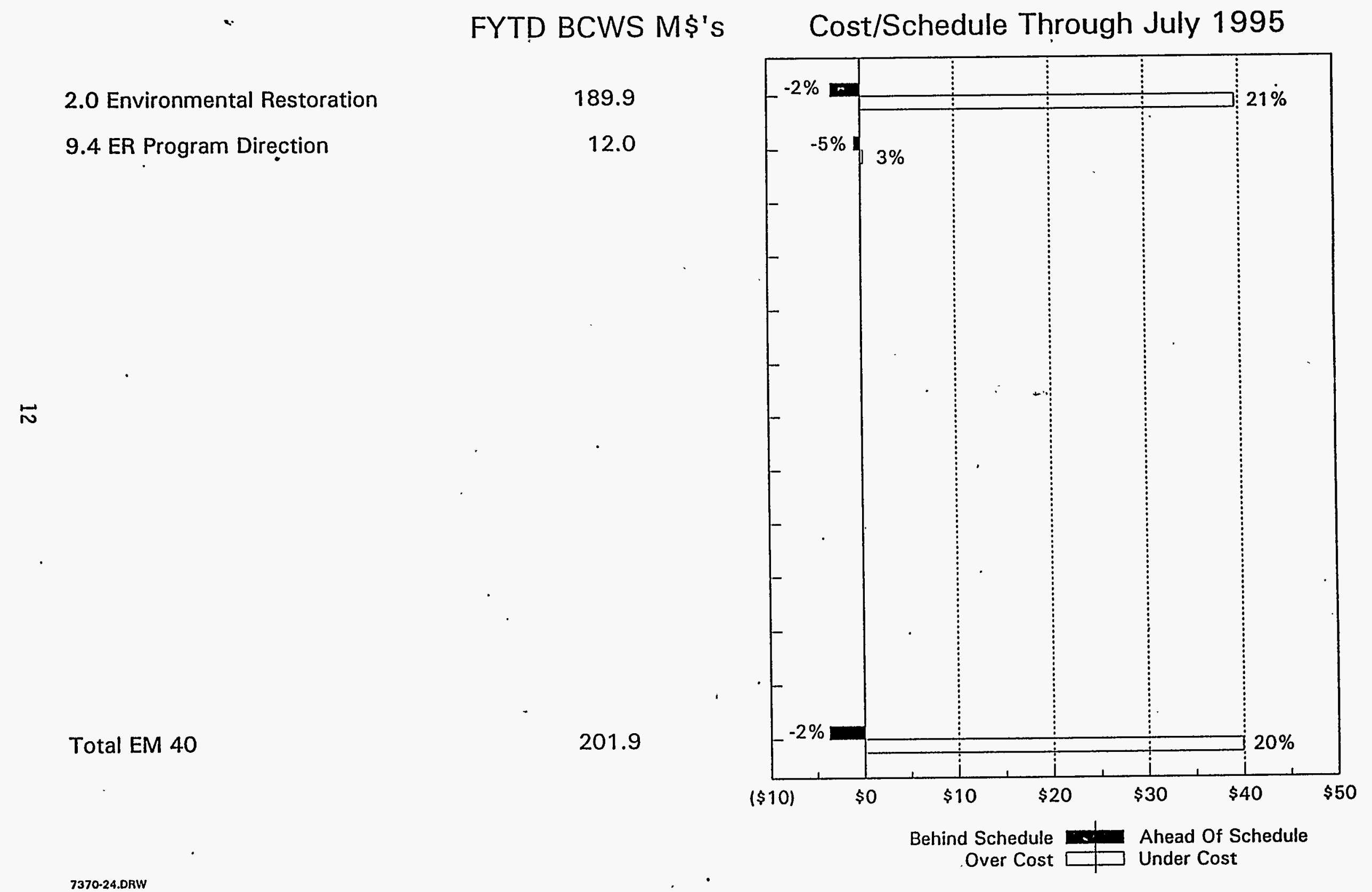

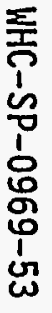




\section{EMI 50 Cost/Schedule Summary Total \$}

\section{FYTD BCWS M\$'s Cost/Schedule Through August 1995}

3.4 Technology Development Support

0

3.5 Technology Development

42.6

$\overleftrightarrow{\omega}$

Total EM 50

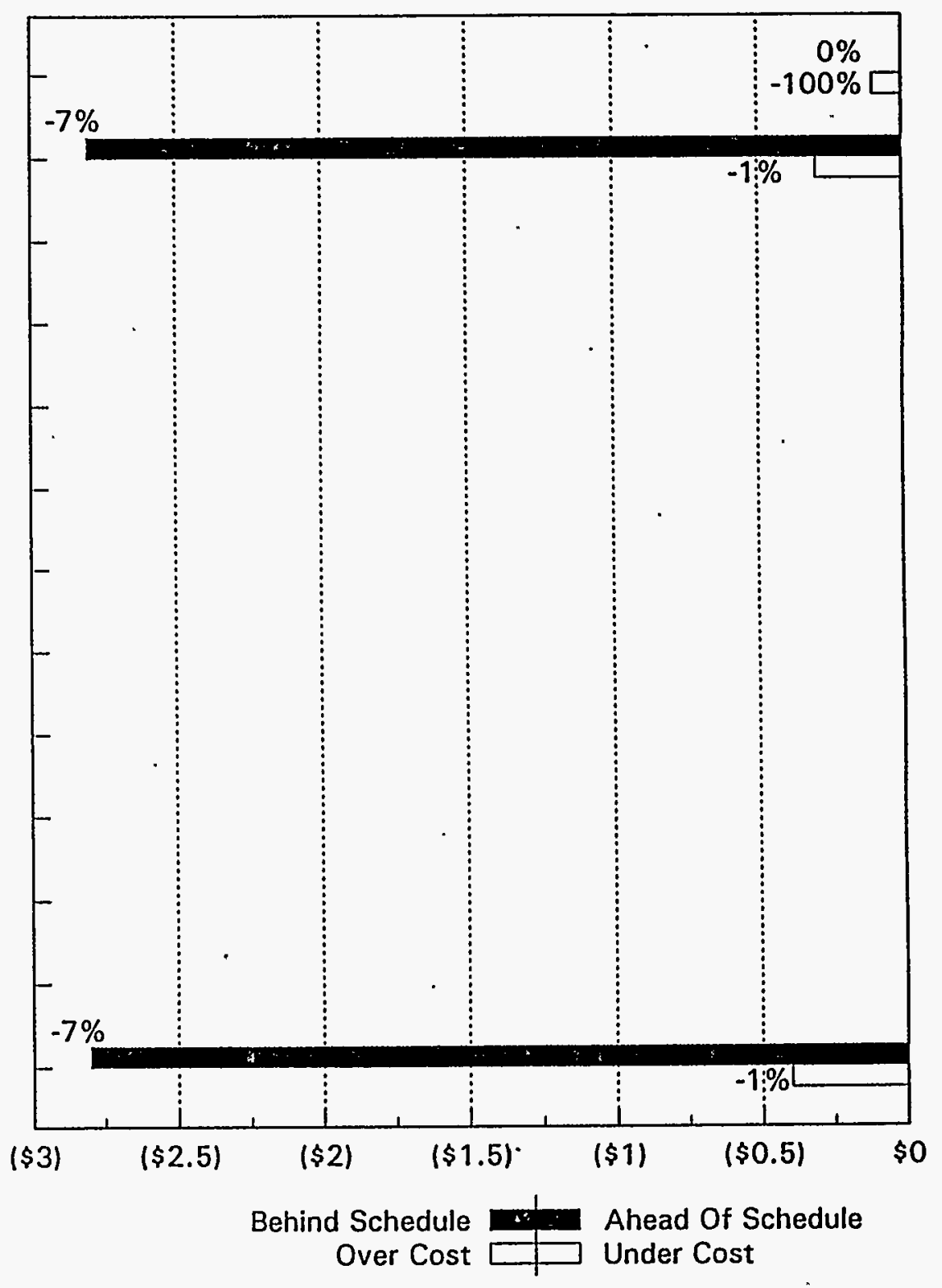

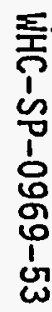




\section{EM 60 Cost/Schedule Summary \\ Total \$}

WBS

7.1 Transition Projects

7.3 Advanced Reactor Transition

7.4.8 Program Direction

7.4.9 Economic Transition

7.5 Landlord

9.6 HO Support To RL

$\ddot{\triangleright}$

\section{FYTD BCWS M\$'s Cost/Schedule Through August 1995}

114.7

51.6

58.1 .

4.2

39.7

0.2

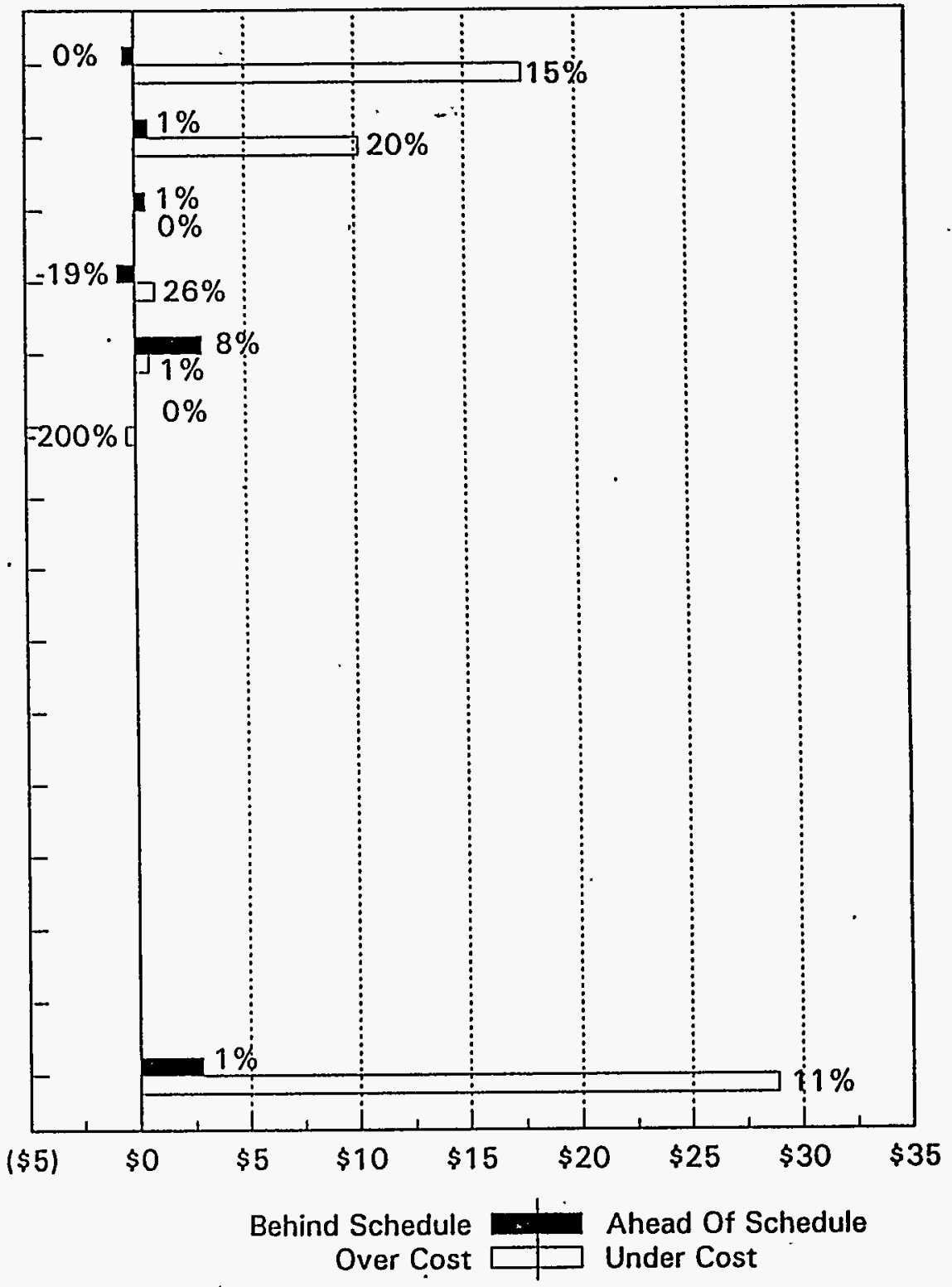

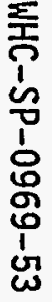




\section{Hanford Operations}

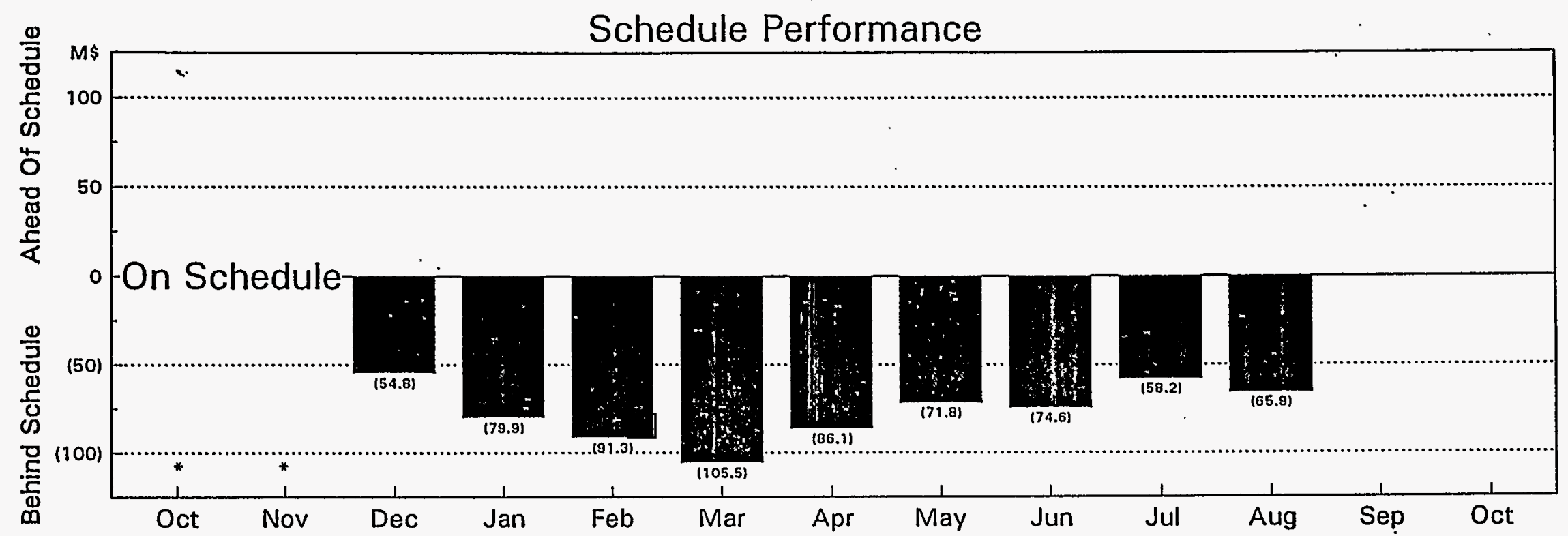

Cost Performance

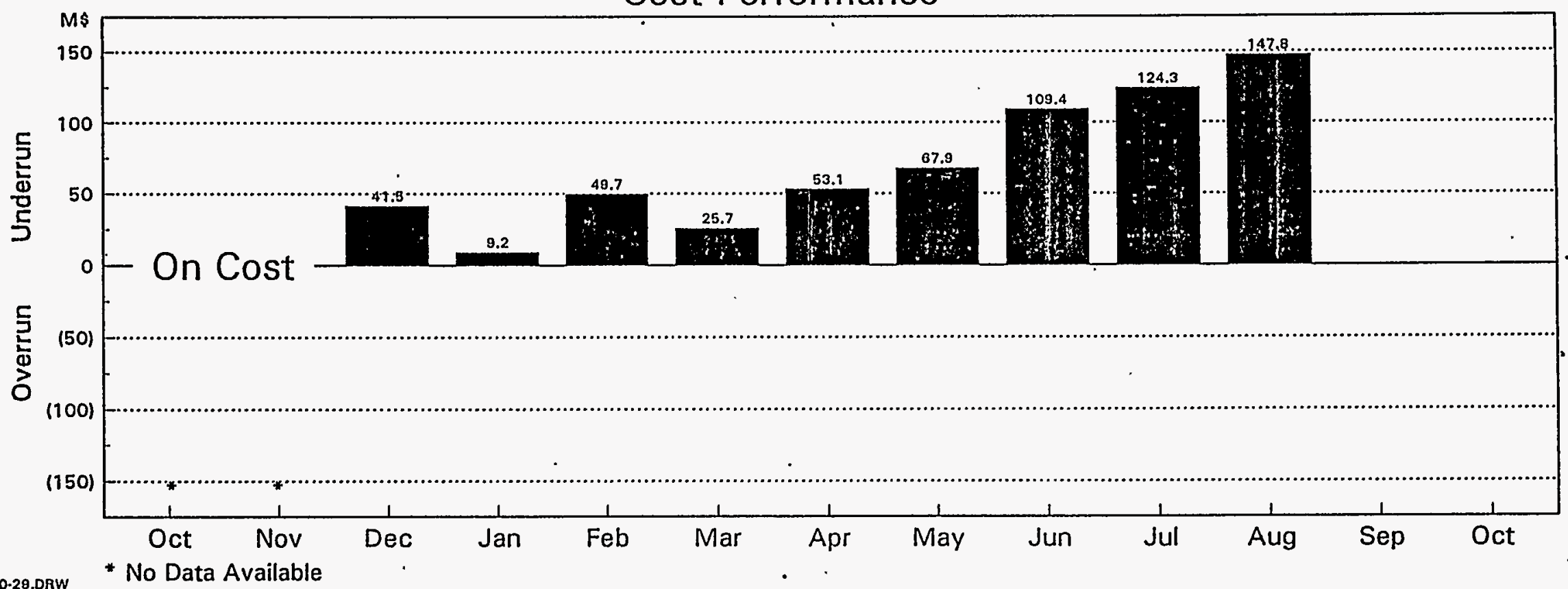


TOTAL EM - ALL FUND TYPES .

AUGUST 1995

( $\$$ In Millions)

9.1/RL Contracting Activitios

3.5.2/Risk Assessment

3.5.3/Outreach

TOTAL EM 10

8.1/Transportation

8.2/HAMMER

8.3/Richland Analytical Sorvicos

8.4/Emergency Management

TOTAL EM 20

1.1/TWRS

1.2.1/Solid Waste

1.2.2/Liquid Waste

1.3.1/Facility Operations

1.4/Spant Nucloar Fuels

$\vec{\sigma}$

CHANGE FROM

ACWP

BCWS BCWP ACWP

$\begin{array}{rrrcccc}2.1 & 2.1 & 2.3 & 0.0 & (0.2) & 2.1 & 0.0 \\ 0.2 & 0.2 & 0.1 & 0.0 & 0.1 & 0.2 & 0.0 \\ 0.1 & 0.0 & 0.3 & (0.1) & (0.3) & 0.1 & 0.0 \\ 2.4 & 2.3 & 2.7 & (0.1) & (0.4) & 2.4 & 0.0 \\ & & & & & & \\ 5.0 & 2.3 & 4.2 & (2.7) & (1.9) & 5.8 & 1.4 \\ 12.7 & 12.7 & 9.2 & 0.0 & 3.5 & 17.6 & (0.1) \\ 2.6 & 2.2 & 1.9 & (0.4) & 0.3 & 2.8 & 0.4 \\ 0.2 & 0.0 & 0.1 & (0.2) & (0.1) & 0.2 & 0.0 \\ 20.5 & 17.2 & 15.4 & (3.3) & 1.8 & 26.4 & 1.7 \\ & & & & & & \\ 528.3 & 473.1 & 430.7 & (55.2) & 42.4 & 595.0 & 4.0 \\ 87.6 & 91.3 & 80.9 & 3.7 & 10.4 & 106.6 & (2.0) \\ 52.1 & 55.0 & 50.8 & 2.9 & 4.2 & 67.0 & 0.1 \\ 31.4 & 30.2 & 30.0 & (1.2) & 0.2 & 35.2 & (3.7) \\ 78.6 & 75.7 & 76.1 & (2.9) & (0.4) & 87.4 & 0.0 \\ 49.4 & 49.2 & 40.9 & (0.2) & 8.3 & 60.2 & (0.7) \\ 8.7 & 8.6 & 5.6 & (0.1) & 3.0 & 10.0 & 0.1 \\ 25.7 & 25.6 & 18.6 & (0.1) & 7.0 & 28.9 & 1.0 \\ 0.5 & 0.5 & 0.4 & 0.0 & 0.1 & 0.6 & 0.0 \\ 41.8 & 37.8 & 35.7 & (4.0) & 2.1 & 47.5 & (0.9) \\ 28.0 & 28.0 & 28.0 & 0.0 & 0.0 & 37.3 & 0.0 \\ 13.0 & 12.6 & 11.3 & (0.4) & 1.3 & 14.8 & 0.1 \\ 2.9 & 2.4 & 1.7 & (0.5) & 0.7 & 3.2 & 0.0 \\ 10.2 & 9.4 & 10.7 & (0.8) & (1.3) & 12.6 & 0.3 \\ 958.2 & 899.4 & 821.4 & (58.8) & 78.0 & 1.106 .3 & (1.7) \\ & & & & & & \\ 189.9 & 186.8 & 147.2 & (3.1) & 39.6 & 238.1 & (10.7) \\ 12.0 & 11.4 & 11.1 & (0.6) & 0.3 & 13.9 & 0.5 \\ 201.9 & 198.2 & 158.3 & (3.7) & 39.9 & 252.0 & (10.2) \\ & & & & & & \\ 0.0 & 0.0 & 0.1 & 0.0 & (0.1) & 0.0 & 0.0 \\ 42.6 & 39.8 & 40.1 & (2.8) & (0.3) & 50.0 & 1.1 \\ 42.6 & 39.8 & 40.2 & (2.8) & (0.4) & 50.0 & 1.1 \\ & & & & & & \\ 268.5 & 271.3 & 242.4 & 2.8 & 28.9 & 305.0 & (7.7) \\ 114.7 & 114.2 & 96.6 & (0.5) & 17.6 & 131.1 & (11.9) \\ 51.6 & 52.2 & 42.0 & 0.6 & 10.2 & 51.6 & 4.3 \\ 58.1 & 58.6 & 58.6 & 0.5 & 0.0 & 72.7 & 0.0 \\ 4.2 & 3.4 & 2.5 & (0.8) & 0.9 & 4.2 & 0.5 \\ 394.7 & 42.7 & 42.1 & 3.0 & 0.6 & 45.2 & (0.6) \\ & 1,428.2 & 1,280.4 & (65.9) & 147.8 & 1,742.1 & (16.8) \\ & & & & & & \end{array}$

$1,428: 2$

$1,8,1 /$ AL Program Diroction

1.8.2/Planning Integration

5.5Mest Valloy

9.X/DOE-HQ ADS

TOTAL EM 30

2.0/Environmental Restoration

9.4/ER Program Direction

TOTAL EM 40

3.4/Technology Development Support

3.5/Technology Development

TOTAL EM 50

7.1/Transition Projocts

7.3/Advanced Reactor Transition

7.4.8/Program Direction

7.4 . $/$ Economic Transition

7.5/Landlord

9.6/HQ Support to RL 
EM EXPENSE COST PERFORMANCE

AUGUST 1995

(\$ In Millions)

9.1/AL Contracting Activities

3.5.2/Risk Assessment

3.5.3/Outreach

TOTALEM 10

\section{1/Transportation}

8.2/HAMMER

8.3/Richland Analytical Sorvicos

8.4/Emergency Management

TOTAL EM 20

\subsection{TWRS}

1.2.1/Solid Waste

1.2.2/Lquid Waste

1.3.1/Facility Operations

1.4/Spent Nuclear Fuels

1.5.1/Analytical Services

1.5.2/Envitonmental Support

1.5.3/RCPA Monitoring

1.5.6 Wasto Minimization

1.7/Science \& Tech Research

1.8.1/RL Program Direction

1.8.2/Planning Integration

5.5Nost Valley

9 XIDOE-HO ADS

TOTAL EM 30

2.0/Environmental Rostoration

9.4/ER Program Direction

TOTAL EM 40

3.4/Technology Dovelopment Support

3.5/Technology Development

TOTALEM 50

7.1/Transition Projects

7.3.1/Advanced Reactor Transition

7.4/Program Diroction

7.4.9/Economic Transition

7.5/Landlord

9.6/HQ Support to RL

TOTALEM 60

TOTAL EM
BCWS BCWP FYTD

$2.1 \quad 2.1$

$\begin{array}{ll}0.1 & 0.0 \\ 2.4 & 2.3\end{array}$

4.8

9.6

9.6
0.2

17.2

412.0

61.6

38.9

78.3

43.8
8.7

8.7
0.9

0.5
39.4

39.4
28.0

13.0

2.9

9.4
789.6

189.9

12.0

0.0

33.2
33.2

111.2

111.2
50.9

50.9
58.1
4.2

4.2
13.4

13.4
0.2

0.2
238.0

$1,282.3$

2.3
0.1
0.3
2.7
4.2
6.3
1.9
0.1
12.5
352.6
48.4
31.7
29.9
75.8
35.9
5.6
17.1
0.4
33.0
28.0
11.3
1.7
9.7
681.1
147.2
11.1
158.3

$0.0 \quad 0.1$

31.0

31.0

109.9

51.5

3.4

3.4
12.9
0.2

236.5

$1,247.8$
93.1

$\begin{array}{rc}2.3 & 0.0 \\ 0.1 & 0.0 \\ 0.3 & (0.1) \\ 2.7 & (0.1) \\ & \\ 4.2 & (2.7) \\ 6.3 & 0.0 \\ 1.9 & (0.4) \\ 0.1 & (0.2) \\ 12.5 & (3.3)\end{array}$

BCWS

FY CHANGE FROM

BCWS PRIORMONTH

$\begin{array}{lll}(0.2) & 2.1 & 0.0\end{array}$

$0.2 \quad 0.0$

$\begin{array}{lll}0.3) & 0.1 & 0.0\end{array}$

$\begin{array}{lll}\text { (2.1) } & 5.6 & 1.4\end{array}$

$3.3 \quad 12.9$

$(0.1) \quad 0.2$

21.5

$\begin{array}{rrr}(13.8) & 45.6 & 463.1 \\ (1.7) & 11.5 & 72.2\end{array}$

72.2
43.9

35.0

87.1

51.8

10.0

24.2
0.6

43.8

37.3

14.8

3.2

11.3

898.3

238.1

13.9
252.0

252.0

0.0

36.8
36.8

0.0
0.4
0.0

0.0

1.8

5.4

0.8
0.0

(3.7)

0.0

(0.5)

0.1
0.0

0.0

(1.0)

0.1

0.1
0.0
0.2

0.2

(10.7)

0.5

(10.2)

0.0

1.1

$\begin{array}{ll}31.9 & (2.2) \\ 32.0 & (2.2)\end{array}$

$(0.9)$
$(1.0)$

126.7

(1.3)
0.6

16.8

10.2
0.0
0.9

50.9

72.7

4.2

15.3
0.2

270.0

$154.2 \quad 1,481.0$

(34.5) 


\section{EM CENRTC PERFORMANCE}

AUGUST 1995

( $\$$ In Millions)

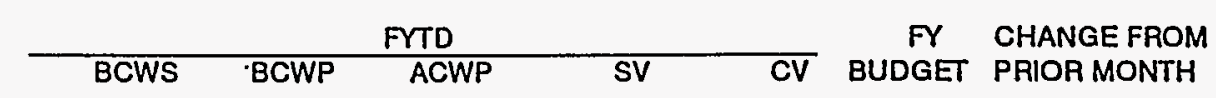

9.1/RL Contracting Activities

3.5.2/Risk Assessment

3.5.3/Outreach

TOTAL EM 10

8.1/Transportation

8.2/HAMMER

8.3/Richland Analytical Services

8.4/Emergency Management TOTAL EM 20

\section{1/TWRS}

1.2.1/Solid Wasto

1.2.2/Liquid Wasto

1.3/Facility Operations

1.4/Spont Nucloar Fuols

1.5.1/Analytical Sorivces

1.5.2/Environmental Support

1.5.3/RCRA Monitoring

1.5.6/Wasto Minimization

1.7.1/Science \& Tech Research

1.8.1/RL Program Direction

1.8.2/Planning Integration

5.5/Wost Valley

9.X/DOE-HQ ADS

TOTAL EM 30

2.0/Environmental Restoration

9.4/ER Program Direction

TOTAL EM 40

3.4/Technology Devolopment Support

3.5/Tochnology Dovolopmont

TOTAL EM 50

7.1/Transition Projects

7.3.1/Advanced Reactor Transition

7.4 Program Direction

7.4.9 Economic Transition

7.5 Landlord

9.6/HQ Support to RL

TOTAL EM 60

\begin{tabular}{|c|c|c|c|c|c|c|}
\hline 0.0 & 0.0 & 0.0 & 0.0 & 0.0 & 0.0 & 0.0 \\
\hline 0.0 & 0.0 & 0.0 & 0.0 & 0.0 & 0.0 & 0.0 \\
\hline 0.0 & 0.0 & 0.0 & 0.0 & 0.0 & 0.0 & 0.0 \\
\hline 0.0 & 0.0 & 0.0 & 0.0 & 0.0 & 0.0 & 0.0 \\
\hline 0.2 & 0.2 & 0.0 & 0.0 & 0.2 & 0.2 & 0.0 \\
\hline 0.0 & 0.0 & 0.0 & 0.0 & 0.0 & 0.0 & 0.0 \\
\hline 0.0 & 0.0 & 0.0 & 0.0 & 0.0 & 0.0 & 0.0 \\
\hline 0.0 & 0.0 & 0.0 & 0.0 & 0.0 & 0.0 & 0.0 \\
\hline 0.2 & 0.2 & 0.0 & 0.0 & 0.2 & 0.2 & 0.0 \\
\hline 34.7 & 38.2 & 34.9 & 3.5 & 3.3 & 40.4 & 1.3 \\
\hline 0.6 & 5.1 & 4.3 & 4.5 & 0.8 & 2.7 & (1.4) \\
\hline 0.2 & 0.3 & 0.3 & 0.1 & 0.0 & 0.2 & 0.0 \\
\hline 0.2 & 0.0 & 0.1 & $(0.2)$ & $(0.1)$ & 0.2 & 0.0 \\
\hline 0.3 & $(0.2)$ & 0.3 & $(0.5)$ & $(0.5)$ & 0.3 & 0.0 \\
\hline 1.7 & 1.8 & 1.2 & 0.1 & 0.6 & 2.5 & 0.0 \\
\hline 0.0 & 0.0 & 0.0 & 0.0 & 0.0 & 0.0 & 0.0 \\
\hline 3.7 & 4.0 & 1.4 & 0.3 & 2.6 & 4.6 & 1.0 \\
\hline 0.0 & 0.0 & 0.0 & 0.0 & 0.0 & 0.0 & 0.0 \\
\hline 0.3 & 0.2 & 0.1 & $(0.1)$ & 0.1 & 1.5 & 0.1 \\
\hline 0.0 & 0.0 & 0.0 & 0.0 & 0.0 & 0.0 & 0.0 \\
\hline 0.0 & 0.0 & 0.0 & 0.0 & 0.0 & 0.0 & 0.0 \\
\hline 0.0 & 0.0 & 0.0 & 0.0 & 0.0 & 0.0 & 0.0 \\
\hline 0.8 & 0.6 & 1.0 & $(0.2)$ & $(0.4)$ & 1.3 & 0.1 \\
\hline 42.5 & 50.0 & 43.6 & 7.5 & 6.4 & 53.7 & 1.1 \\
\hline 0.0 & 0.0 & 0.0 . & 0.0 & 0.0 & 0.0 & 0.0 \\
\hline 0.0 & 0.0 & 0.0 & 0.0 & 0.0 & 0.0 & 0.0 \\
\hline$\cdot 0.0$ & 0.0 & 0.0 & 0.0 & 0.0 & 0.0 & 0.0 \\
\hline 0.0 & 0.0 & 0.0 & 0.0 & 0.0 & 0.0 & 0.0 \\
\hline 9.4 & 8.8 & 8.2 & $(0.6)$ & 0.6 & 13.2 & 0.0 \\
\hline 9.4 & 8.8 & 8.2 & $(0.6)$ & 0.6 & 13.2 & 0.0 \\
\hline 1.2 & 2.0 & 0.9 & 0.8 & 1.1 & 1.5 & 0.0 \\
\hline 0.1 & 0.1 & 0.1 & 0.0 & 0.0 & 0.1 & 0.0 \\
\hline 0.0 & 0.0 & 0.0 & 0.0 & 0.0 & 0.0 & 0.0 \\
\hline 0.0 & 0.0 & 0.0 & 0.0 & 0.0 & 0.0 & 0.0 \\
\hline 4.4 & 5.8 & 5.5 & 1.4 & 0.3 & 4.9 & 0.3 \\
\hline 0.0 & 0.0 & 0.0 & 0.0 & 0.0 & 0.0 & 0.0 \\
\hline 5.7 & 7.9 & 6.5 & 2.2 & 1.4 & 6.5 & 0.3 \\
\hline 57.8 & 66.9 & 58.3 & 9.1 & 8.6 & 73.6 & 1.4 \\
\hline
\end{tabular}




\section{EM GPP/LINE ITEM PERFORMANCE}

\section{AUGUST 1995}

( $\$$ In Millions)

BCWS

FY CHANGE FROM

BCWS BCWP ACWP SV

9.1/RL Contracting Activities

3.5.2/Risk Assessment

3.5.3/Outreach

Total EM 10

8.1/Transportation

8.2/HAMMER

8.3/Aichland Analytical Services

8,4/Emergency Managomont TOTAL EM 20

\section{1/TWRS}

1.2.1/Solid Wasto

1.2.2/Liquid Wasto

1.3.1/Facility Oporations

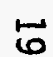

0.0

\begin{tabular}{|c|c|}
\hline 0.0 & 0.0 \\
\hline 0.0 & 0.0 \\
\hline 0.0 & 0.0 \\
\hline 0.0 & 0.0 \\
\hline 0.0 & 0.0 \\
\hline 3.1 & 2.9 \\
\hline 0.0 & 0.0 \\
\hline 0.0 & 0.0 \\
\hline 3.1 & 2.9 \\
\hline
\end{tabular}

81.6

25.4

$36.7 \quad 43.2$

36.7

26.3

16.6
0.0

0.0

43.2
28.2

28.2

18.8

0.0

\section{(6.5)}

91.5

$\begin{array}{ll}(1.9) & 31.7 \\ & \end{array}$

0.0

0.0

1.5.1/Site Support

1.5.2/Environmental Support

1.5.3/RCRA Monitoring

1.5.6/Waste Minimization

1.7.1/Research

1.8.1/RL Program Direction

1.8.2 Planning Integration

5.5/Wost Valley

9.0/DOE-HQ ADSs

TOTAL EM 30

2.0/Environmental Restoration

9.4/ER Program Direction

TOTAL EM 40

3.4/Tochnology Dovolopment Support 3.5/Tochnology Devolopment

TOTAL EM 50

7.1/Transition Projects

7.3.1/Advanced Roactor Transition

7.4/Program Diroction

7.4.9/Economic Transition

7.5/Landlord

9.6/HQ Support to RL

TOTAL EM 60

TOTAL

3.9

0.0

0.1
0.0

0.0
$(0.1)$

$(0.1)$

0.0
0.0

0.0
0.0

0.0
0.0

0.0
83.5

126.1

0.0

0.0

0.0

0.0

0.0
0.0

2.3

0.6
0.0

0.0

0.0

21.9

0.0
24.8

3.8

0.0

0.1

0.0

(1.9) 31.7 


\section{TWRS ALL FUND TYPES COST PERFORMANCE BY.ADS}

$\therefore$

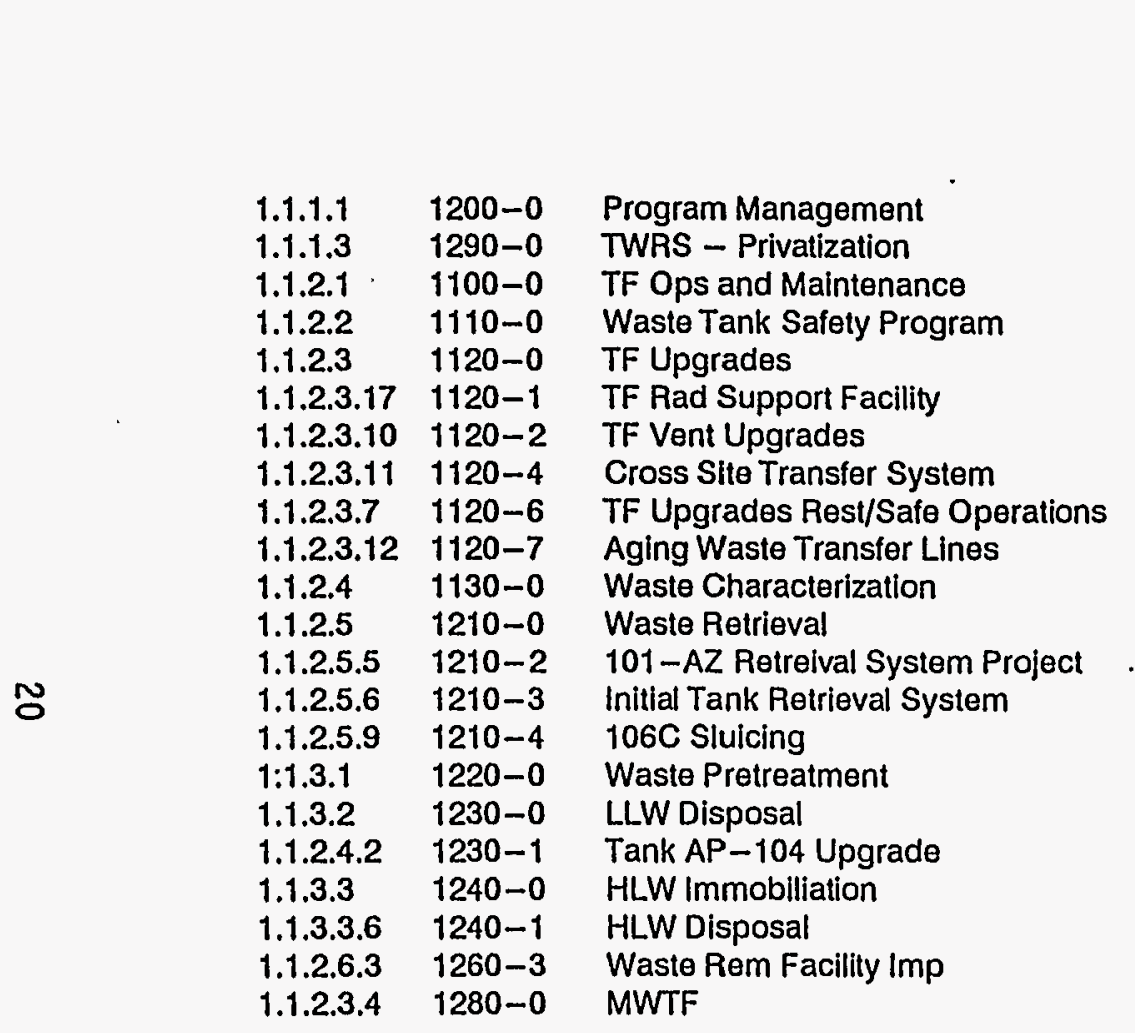

TOTAL

\section{AUGUST 1995 \\ ( $\$$ In Millions)}

BCWS BCWP FYTD

$\begin{array}{rrr}49.4 & 47.8 & 41.8 \\ 10.5 & 10.5 & 9.9 \\ 130.9 & 130.5 & 111.9 \\ 50.2 & 55.0 & 47.4 \\ 21.5 & 17.3 & 18.9 \\ 0.0 & 0.0 & 0.0 \\ 11.3 & 9.6 & 10.9 \\ 3.1 & 4.2 & 3.7 \\ 7.1 & 4.9 & 5.0 \\ 0.5 & 0.4 & 0.5 \\ 75.6 & 75.1 & 68.5 \\ 7.0 & 6.7 & 6.5 \\ 4.3 & 4.7 & 6.3 \\ 3.6 & 3.6 & 3.3 \\ 13.7 & 12.6 & 12.7 \\ 19.0 & 17.4 & 15.2 \\ 31.5 & 30.1 & 24.6 \\ (1.1) & (0.3) & 0.0 \\ 13.5 & 12.0 & 9.5 \\ 6.2 & 5.0 & 6.0 \\ 0.0 & 0.0 & 0.0 \\ 70.5 & 26.0 & 28.1 \\ & & \\ 528.3 & 473.1 & 430.7\end{array}$

FY BCWS

FY CHANGE FROM CV BCWS PRIOR MONTH

$\begin{array}{ccrc}(1.6) & 6.0 & 53.3 & 2.0 \\ 0.0 & 0.6 & 14.5 & 14.5 \\ (0.4) & 18.6 & 146.5 & (1.4) \\ 4.8 & 7.6 & 53.2 & (1.1) \\ (4.2) & (1.6) & 23.4 & (1.3) \\ 0.0 & 0.0 & 0.0 & 0.0 \\ (1.7) & (1.3) & 11.8 & (0.0) \\ 1.1 & 0.5 & 3.5 & (2.0) \\ (2.2) & (0.1) & 7.2 & 0.0 \\ (0.1) & (0.1) & 0.6 & (0.5) \\ (0.5) & 6.6 & 91.9 & 0.1 \\ (0.3) & 0.2 & 7.9 & (3.9) \\ 0.4 & (1.6) & 5.7 & 2.5 \\ 0.0 & 0.3 & 4.5 & 0.8 \\ (1.1) & (0.1) & 16.1 & 0.1 \\ (1.6) & 2.2 & 22.8 & (1.3) \\ (1.4) & 5.5 & 33.6 & (1.0) \\ 0.8 & (0.3) & (1.1) & 0.0 \\ (1.5) & 2.5 & 15.1 & (3.1) \\ (1.2) & (1.0) & 7.2 & 0.0 \\ 0.0 & 0.0 & 0.0 & 0.0 \\ (44.5) & (2.1) & 77.3 & (0.4) \\ & & & \\ (55.2) & 42.4 & 595.0 & 4.0\end{array}$




\section{SCHEDULE VARIANCE}

- Hanford schedule performance did not improve in August

$\begin{array}{ll}\text { DECEMBER } & (\$ 54.8 \mathrm{M})(14 \%) \\ \text { JANUARY } & (\$ 79.9 \mathrm{M})(15 \%) \\ \text { FEBRUARY } & (\$ 91.3 \mathrm{M})(13 \%) \\ \text { MARCH } & (\$ 105.5 \mathrm{M})(13 \%) \\ \text { APRIL } & (\$ 86.1 \mathrm{M})(9 \%) \\ \text { MAY } & (\$ 71.8 \mathrm{M})(7 \%) \\ \text { JUNE } & (\$ 74.6 \mathrm{M})(6 \%) \\ \text { JULY } & (\$ 58.2 \mathrm{M})(4 \%) \\ \text { AUGUST } & (\$ 65.9 \mathrm{M})(4 \%)\end{array}$

- The majority of the schedule variance is attributed to EM-30 - specifically TWRS. The biggest contributors to the TWRS schedule variance include:

- DOE-HQ delays in approving KD-0 for Project W-314 (Tank Farm Upgrades, ADS 1120-6; -\$2.0M).

- MWTF is still part of TWRS baseline (ADS $1280-0 ;-\$ 44.5 \mathrm{M}$ ) 


\section{COST VARIANCE}

- Hanford cost performance continues to underrun and is attributed to achievement of productivity goals; it should continue for the remainder of the year

DECEMBER
JANUARY
FEBRUARY
MARCH
APRIL
MAY
JUNE
JULY
AUGUST

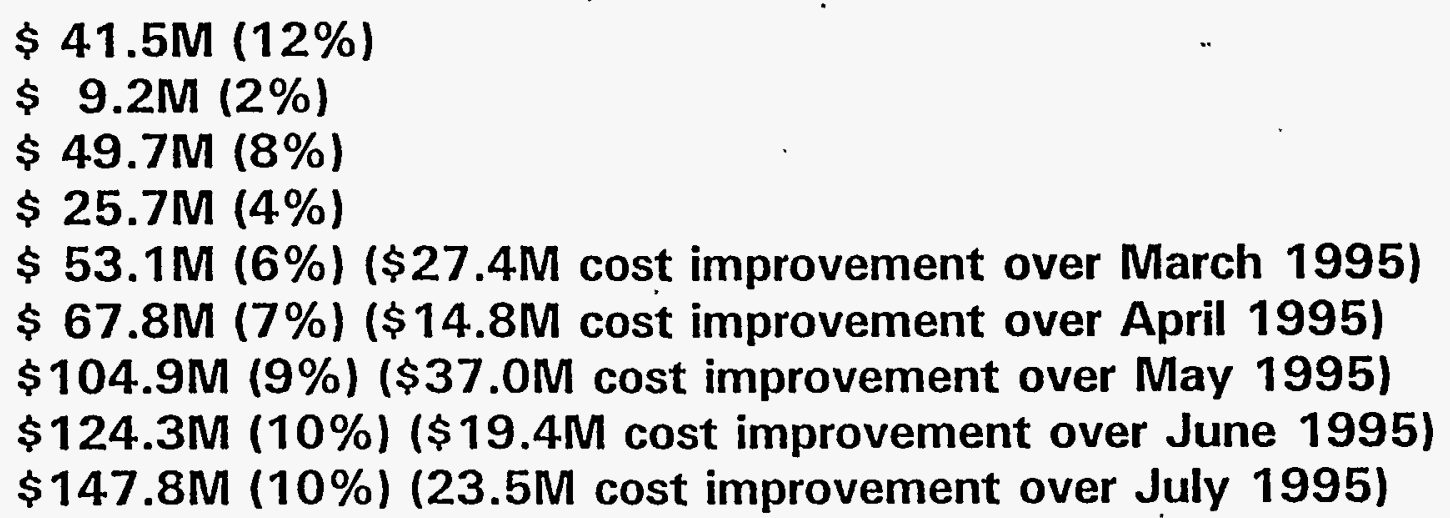

- Major contributors to the underrun

EM-30 \$78.0M underrun

- Process improvements/efficiencies

- Elimination of low-value work

- Workforce reductions

- EM-40 \$39.9M underrun

- Automation and more efficient use of resources

- Productivity improvements 


\section{COST VARIANCE}

(Continued)

- EM-60 \$28.9M underrun

- Process improvements/efficiencies

- Elimination of low-value work

- Workforce reductions 

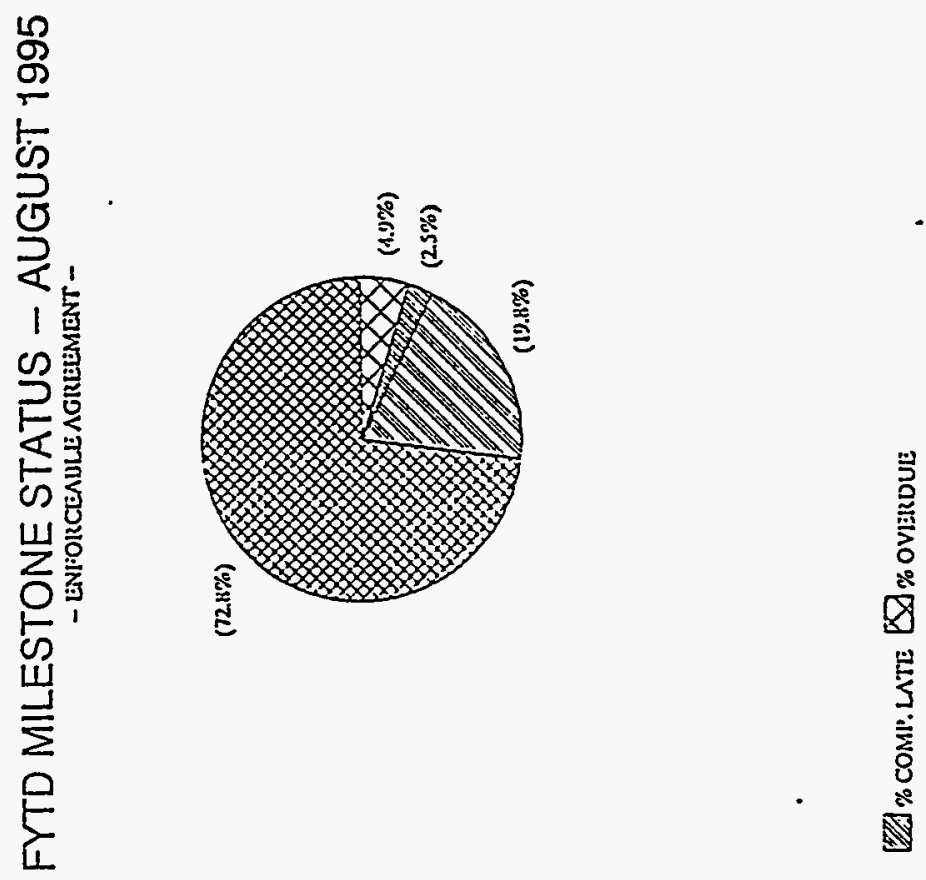

\%

向

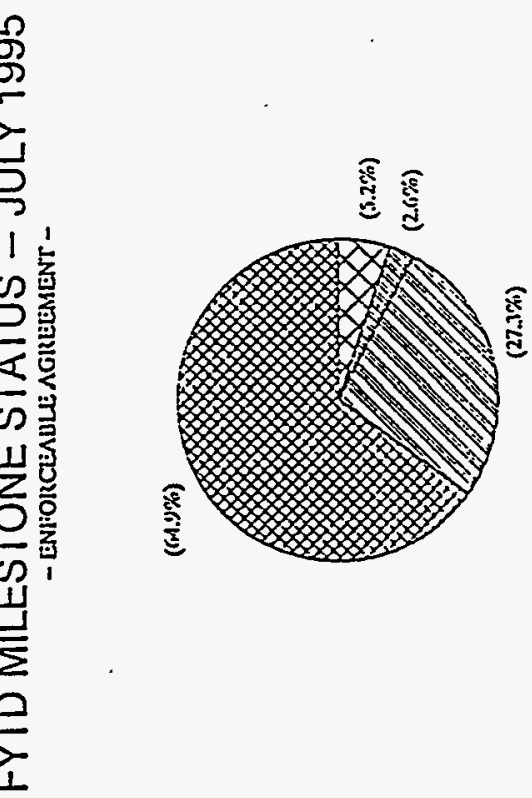

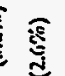

承

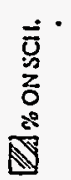




\section{FY 1995 MILESTONE STATUS - ENFORCEABLE AGREEMENT AUGUST 1995}

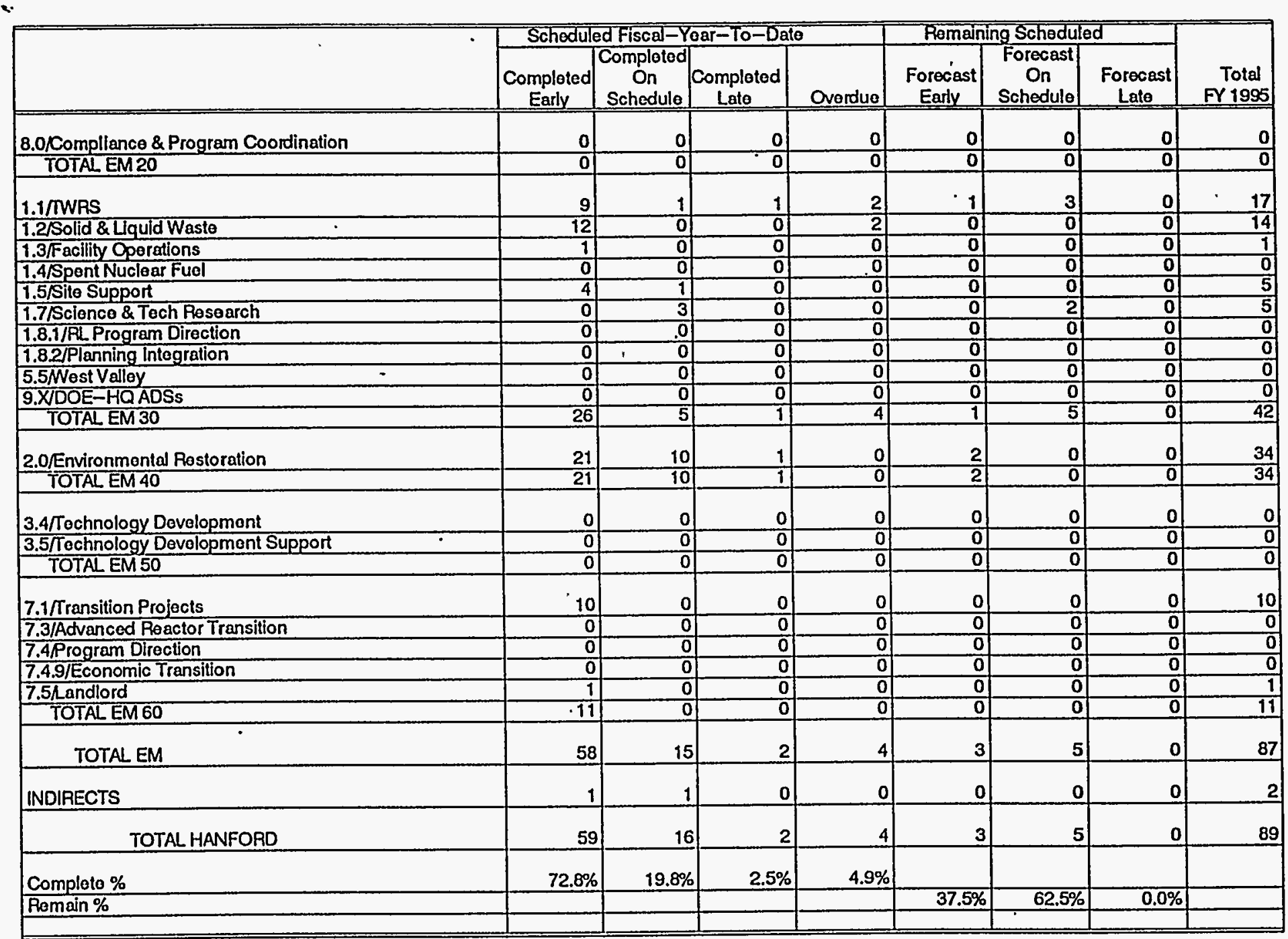




\section{MILESTONE EXCEPTIONS - ENFORCEABLE AGREEMENT MILESTONES}

\section{WBS \\ TYPE \\ MILESTONE \\ DUE BUT NOT COMPLETE}

1.1

\author{
TPA-I W-314B DST Ventilation \\ Upgrades CDR \\ (ADS 1120) (M-43-02A)
}

\section{FORECAST \\ COMP.}

\section{CAUSE/IMPACT/RECOVERY PLAN}

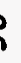

1.1 TPA-1 W-314A Tank Farm Instrumentation Upgrades CDR (ADS 1120)

(M-43-04A)
$05 / 95 \quad 05 / 96$

Cause: Delay in approval of KD-O.

Impact: Project has been delayed approximately one year. Impacts being assessed.

Recovery Plan: Approval of KD-0 was received in February 1995 (approval was scheduled for July 1994); work initiated. Change request extending the milestone date was disapproved. A draft Tri-Party Agreement change request will be submitted with the TWRS MYPP documenting the impacts to the Tri-Party Agreement milestones and will parallel the June 15, 1995, recovery plan submitted to Ecology.

05/95 05/96 Same as above. 


\title{
MILESTONE EXCEPTIONS - ENFORCEABLE AGREEMENT MILESTONES
}

\author{
$\begin{array}{llcc} & & \text { BASELINE } & \text { FORECAST } \\ \text { WBS TYPE } & \text { MILESTONE } & \text { DATE } & \text { COMP. }\end{array}$ \\ CAUSE/IMPACT/RECOVERY PLAN
}

$1.2 \quad$ TPA-1 Initiate Operations - 200

$06 / 95 \quad 03 / 96$

Cause: The 200 Area ETF construction Area ETF

(M-17-14) (ADS 2300) delay has impacted this milestone. Impact: Impacts are being reviewed with regulators and $\mathrm{RL}$. Forecast completion date is based on those discussions. Recovery Plan: The Tri-Parties have been meeting since February 1995 to discuss the strategy for proceeding with these milestones. All parties agreed to: 1) reword M-17-00A to allow for temporary storage of process condensate stream in the LERF Basins until BAT/AKART

implementation occurred; and, 2) RL will withdraw the dispute on extending $M-17-14$ and $M-17-29$ completion dates and these two interim milestones would be missed (they will be completed during the first quarter of FY 1996).

for 242-A Evaporator Process Condensate (M-17-29) (ADS 2300) 

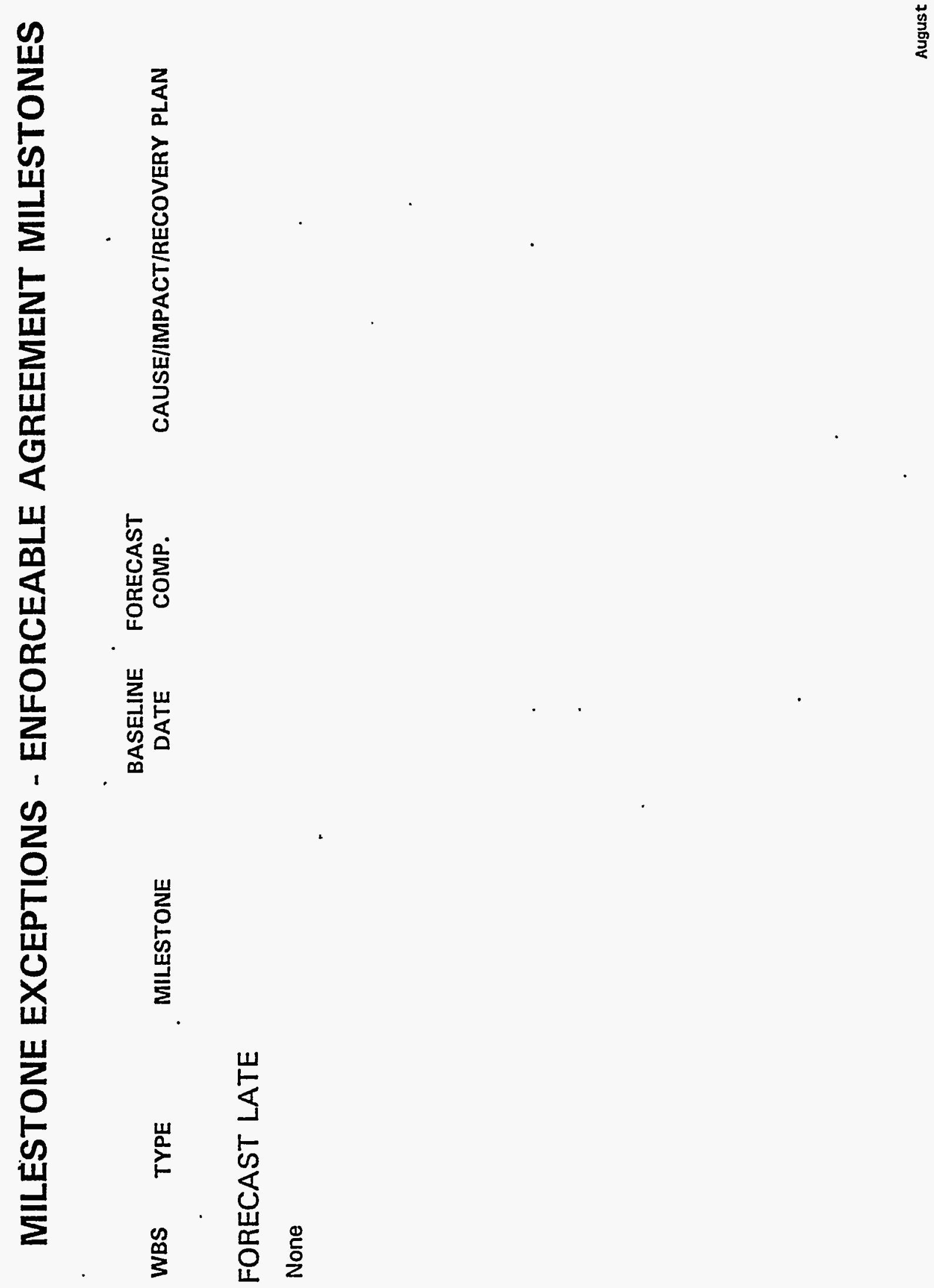


\section{DISTRIBUTION}

\section{Number of copies}

\section{OFFSITE}

U.S. Department of Enerqy - Headquarters 1000 Independence Ave., S.W.

Washington, D.C. 20585

Steven R. Woodbury (EH-411)

Gary 0. Roberson (EM-36)

J. V. Antizzo (EM-36)

1

B\&W Nuclear Environmentz? Services. Inc.

P.0. Box 10548

2200 Langhorne Road

Lynchburg, VA 24506-0548

Gary W. Jinith

1

Benton County Treasurer's Office

P.0. Box 630

Prosser, WA 99350-0630

Scott Holt

2

Confederated Tribes of the Umatilla

Indian Reservation

P.0. Box 638

Pendleton, OR 97801

Elwood Patawa

1 Department of Health

Nuclear Safety Section

1511 - 3rd Ave., Suite 700

Seattle, WA 98101

Robert R. Mooney

6

DNFSB

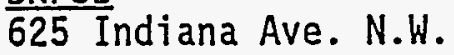

Suite 700

Washington, D.C. 20004

Dermot M. Winters

1

Enserch Environmental Corporation

1981 Snyder Road, Suite 3

Richland, WA 99352

Glenn Cox

$$
\text { Distr-1 }
$$




\section{WHC-SP-0969-53 \\ DISTRIBUTION (cont)}

Number of copies

OFFSITE

1

Golder Associates, Inc.

1933 Jadwin Ave., Suite 125

Richland, WA 99352

Ken Moser

1

Hanford Advisory Board

Gordon Rodgers

1108 Road 36

Pasco, WA 99301

1

Oregon State University

Graduate School

Administrative Services, A-300

Corvallis, OR 97731

John Ringle

1

Physicians for Social Responsibilities

030 S.W. Ridge Drive

Portland, $O R$ 97219-6566

Richard Belsey, M.D.

1

PPC Environmental Management

1411 - 4th Ave., Suite 720

Seattle, WA 98101

Jeff Ross

1

$\underline{\text { SAIC }}$

$\frac{\text { AAIC }}{1845}$ Terminal Drive

Richland, WA 99352

Jerry White

1

Washington Physicians for Social Responsibility 4534 1/2 University Way N.E.

Seattie, WA 98105

Mark Bigelow 


\section{DISTRIBUTION (cont)}

Number of copies

OFFSITE

1

Montgomery Watson

1201 Jadwin Ave., Suite 202

Richland, WA 99352

Kevin E. Kelly

ONSITE

1

U.S. Department of Energy, Richland Operations office

Public Reading Room

H2-53

1

U.S. Environmental Protection Agency

D. R. Einan

B5-01

3

Kaiser Engineers Hanford

K. J. Dempsey

R. E. Tiller

T. L. Watson

E6-61

E6-61

S3-10

11

Westinghouse Hanford Company

C. S. Wilson (7)

Central Files

Correspondence Control

H6-08

L8-04

OSTI (2)

A3-01

A3-36

Distr-3 